\title{
A Peculiar Interacting Be Star Binary in the Small Magellanic Cloud*
}

\author{
Ronald E. Mennickent ${ }^{1}$, Thomas Rivinius ${ }^{2}$, Lydia Cidale ${ }^{3,4,5}$ Igor Soszyńsk $^{6}$, and J. G. Fernández-Trincado ${ }^{1,7}$ \\ ${ }^{1}$ Departamento de Astronomía, Casilla 160-C, Universidad de Concepción, Chile; rmennick@udec.cl \\ ${ }^{2}$ European Organization for Astronomical Research in the Southern Hemisphere, Casilla 19001, Santiago 19, Chile \\ ${ }^{3}$ Facultad de Ciencias Astronómicas y Geofísicas, Universidad de La Plata, Argentina \\ ${ }^{4}$ Instituto de Astrofísica de La Plata, CONICET-UNLP, Argentina \\ ${ }^{5}$ Instituto de Física y Astronomía, Facultad de Ciencias, Universidad de Valparaíso, Av. Gran Bretaña 1111, Casilla 5030, Valparaíso, Chile \\ ${ }^{6}$ Warsaw University Observatory, Al. Ujazdowskie 4, 00-478 Warszawa, Poland \\ ${ }^{7}$ Institut Utinam, CNRS UMR6213, Univ. Bourgogne Franche-Comté, OSU THETA, Observatorie de Besançon, BP 1615, 25010 Besançon Cedex, France \\ Received 2018 April 27; accepted 2018 June 5; published 2018 July 24
}

\begin{abstract}
We find that the emission-line object OGLEJ005039.05-725751.4, a member of the cluster OGLE-CL SMC 64, exhibits a peculiar light curve pattern repeating with a recurrence time of 141.45 days. The light curve resembles periodic outbursts with a duty cycle of $20 \%$. A second long cycle of 2500 days is also detected in the photometric data set. Two X-SHOOTER spectra obtained at minimum and maximum reveal a Be star dominating at minimum light resembling the Classical Be star 48 Lib. The larger $\mathrm{H} \alpha$ emission, the stronger $\mathrm{NaD}$ absorption and the appearance of emission in the infrared $\mathrm{Ca}$ II triplet at maximum, might indicate periodic mass transfer in a complex binary system.
\end{abstract}

Key words: (stars:) binaries (including multiple): close - (stars:) binaries: spectroscopic - (stars:) circumstellar matter - stars: emission-line - Be - stars: evolution - stars: variables: general

Online material: color figures

\section{Introduction}

The object OGLEJ005039.05-725751.4 $(V=17.211 \mathrm{mag}$, $\left.\alpha_{2000}=00: 50: 39.1630, \delta_{2000}=-72: 57: 51.239\right)^{8}$ is a member of the Small Magellanic Cloud, and it was classified as a Be star candidate, based on optical colors and light curve variability by Mennickent et al. (2002). Later, and consistently with this classification, $\mathrm{H} \alpha$ emission was reported in a slitless survey by Martayan et al. (2010). The object is a member of the cluster OGLE-CL SMC 64 (Bica \& Dutra 2000), and possibly is the same object catalogued as 2MASS J00504006-7257492 (SSTISAGEMA J005040.07-725749.3) with coordinates $\alpha_{2000}=00: 50: 40.067, \delta_{2000}=-72: 57: 49.21$ and labeled as a possible red giant branch star in the SIMBAD database.

The light curve of OGLEJ005039.05-725751.4 shows an extremely rare, strict repeatability of a Be star outburst like brightening with a period of 141.45 days. In order to investigate the nature of this object we obtained two spectra at maximum and minimum light. The analysis of these spectra and survey light curve is reported in this paper. A finding chart for the star and its surrounding stellar field is shown in Figure 1.

The paper is organized as follows. In Section 2, we introduce the photometric data sets used in our analysis; details of our

\footnotetext{
* Based on the ESO proposal 098.D-0099(A).

8 http://simbad.u-strasbg.fr/simbad/
}

spectroscopic observations are given in Section 3; in Section 4, we present our results including the light curve analysis and the study of the spectroscopic data; in Section 5, a discussion is provided along with a possible interpretation for the system; and finally our conclusions are given in Section 6.

\section{Photometric Data}

The photometric time-series data analyzed in this paper were taken from the OGLE project databases. We included OGLE-II data (Szymanski 2005) $)^{9}$ and OGLE-III/IV data. ${ }^{10}$ The OGLE-IV project is described by Udalski et al. (2015). The whole data set consists of $1501 \mathrm{I}$-band magnitudes and $143 \mathrm{~V}$-band magnitudes taken during a time interval of 17.08 years. A summary of these data sets is given in Table 1 .

We considered to study the spectral energy distribution of this object with broadband photometry provided by the VizieR photometric tool. ${ }^{11}$ This online tool extracts the magnitudes published in surveys and catalogues, based in a search performed around a given location, considering a searching radius. We find that the scatter shown by the fluxes is quite large, more than expected from the variability of the object, and unfortunately inadequate for our study. It is possible that the

\footnotetext{
9 http://ogledb.astrouw.edu.pl/ ogle/photdb/

${ }_{11}^{10}$ OGLE-III/IV data kindly provided by the OGLE team.

11 http://vizier.u-strasbg.fr/vizier/sed/doc/
} 


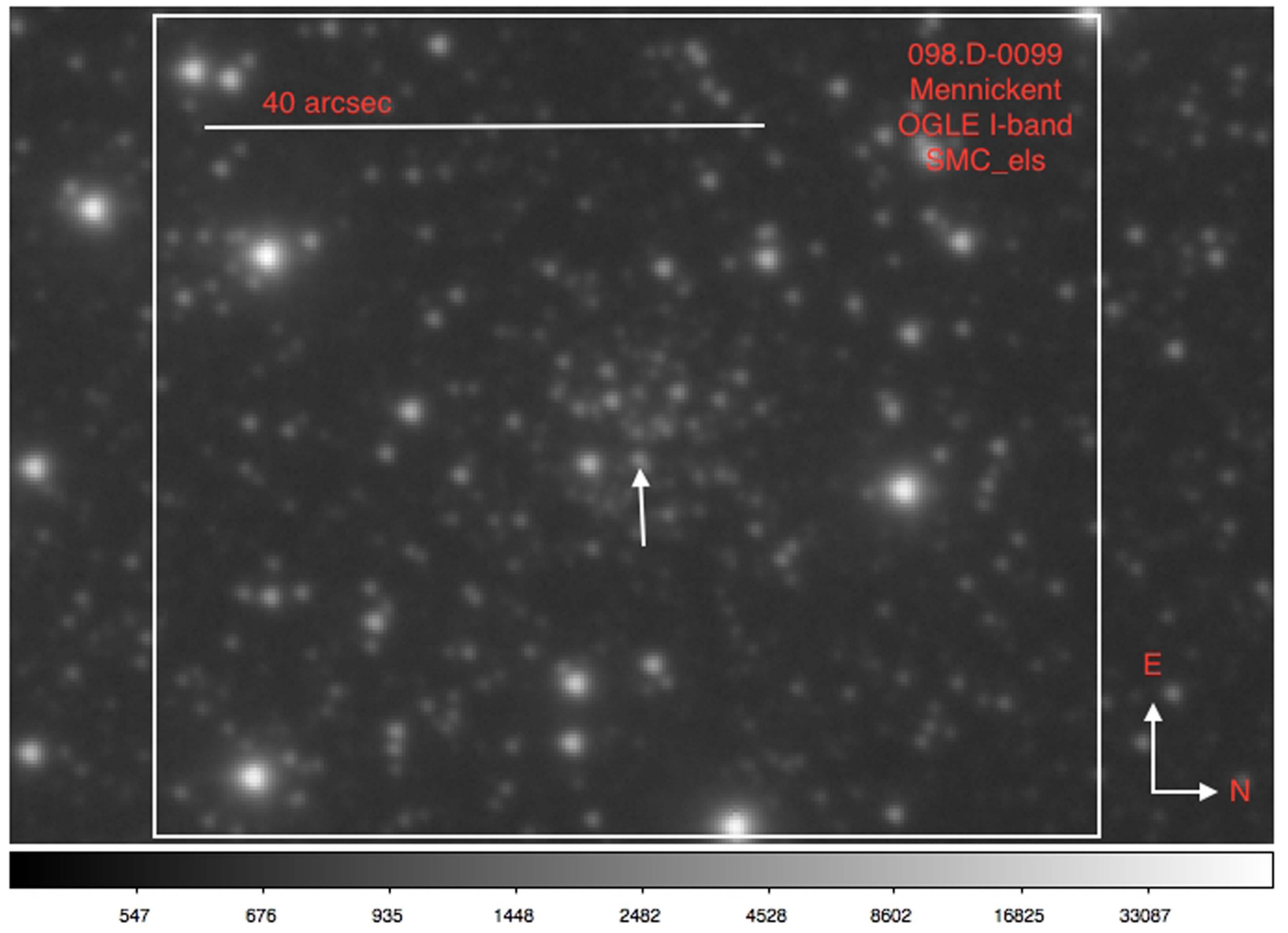

Figure 1. Stellar field around OGLEJ005039.05-725751.4 according to an OGLE I-band image. OGLEJ005039.05-725751.4 is shown by the arrow at the center of the image.

Table 1

Summary of Survey Photometric Observations. The Number of Measurements, Starting and Ending Times for the Series and Average Magnitude and Standard Deviation (in Magnitude) are Given. The Zero Point of HJD is 2450000. Single-point Uncertainties in the $I$ band and $V$ band are between 4 and 6 mmag

\begin{tabular}{lrrcccc}
\hline \hline Database & \multicolumn{1}{c}{$\mathrm{N}$} & \multicolumn{1}{c}{ HJD $_{\text {start }}$} & HJD $_{\text {end }}$ & Mag. & Std. & Band \\
\hline OGLE-II & 332 & 466.5440 & 1871.7550 & 16.543 & 0.126 & $I$ \\
OGLE-III & 732 & 2085.9091 & 4954.8884 & 16.864 & 0.174 & $I$ \\
OGLE-IV & 437 & 5346.9189 & 6704.5196 & 16.909 & 0.143 & $I$ \\
OGLE-II & 44 & 466.5830 & 1543.6290 & 17.037 & 0.056 & $V$ \\
OGLE-III & 53 & 3326.5608 & 4954.8940 & 17.053 & 0.056 & $V$ \\
OGLE-IV & 46 & 5391.9156 & 6601.5731 & 17.053 & 0.061 & $V$ \\
\hline
\end{tabular}

presence of several nearby objects in the crowded field is the origin of this scatter, considering that automatic photometric algorithms might fail in such circumstances.

\section{Spectroscopic Data}

We obtained two spectra for OGLEJ005039.05-725751.4 during the nights of 2017 June 2-3 and July 12-13 with the
Table 2

Summary of X-SHOOTER Spectroscopic Observations. The Heliocentric Julian day $\left(\mathrm{HJD}^{\prime} \equiv \mathrm{HJD}-2457\right.$ 900) at Mid-exposure and Wavelength Range are given; $R$ is Resolving Power and Signal-to-noise Ratio (S/N)

Measured Around 400 and $680 \mathrm{~nm}$. $\Phi$ Refers to the Phase to the Ephemerides given by Equation (1)

\begin{tabular}{lrccccc}
\hline \hline $\begin{array}{l}\text { Night } \\
(2017)\end{array}$ & $\begin{array}{c}\Delta \lambda \\
(\mathrm{nm})\end{array}$ & $R$ & $\begin{array}{c}\text { Exptime } \\
(\mathrm{s})\end{array}$ & $\mathrm{S} / \mathrm{N}$ & HJD $^{\prime}$ & $\Phi$ \\
\hline 2/3-Jun & $534-1020$ & 5400 & 1260 & 35 & 07.90506 & 0.753 \\
2/3-Jun & $299-556$ & 7400 & 1161 & 55 & 07.90454 & 0.753 \\
12/13-Jul & $534-1020$ & 5400 & 1260 & 10 & 47.77828 & 0.035 \\
12/13-Jul & $299-556$ & 7400 & 1161 & 50 & 47.77776 & 0.035 \\
\hline
\end{tabular}

ESO X-SHOOTER spectrograph. This three-arms echelle spectrograph is located on Unit Telescope 2 (UT2; Kueyen) of the Very Large Telescope (VLT) at the Paranal Observatory, Chile, and provides intermediate resolution spectroscopy across a wide wavelength range, from the ultraviolet (UV) to the nearinfrared (NIR). Our observing setup was optimized to get good 


\section{HJD - 2450000}
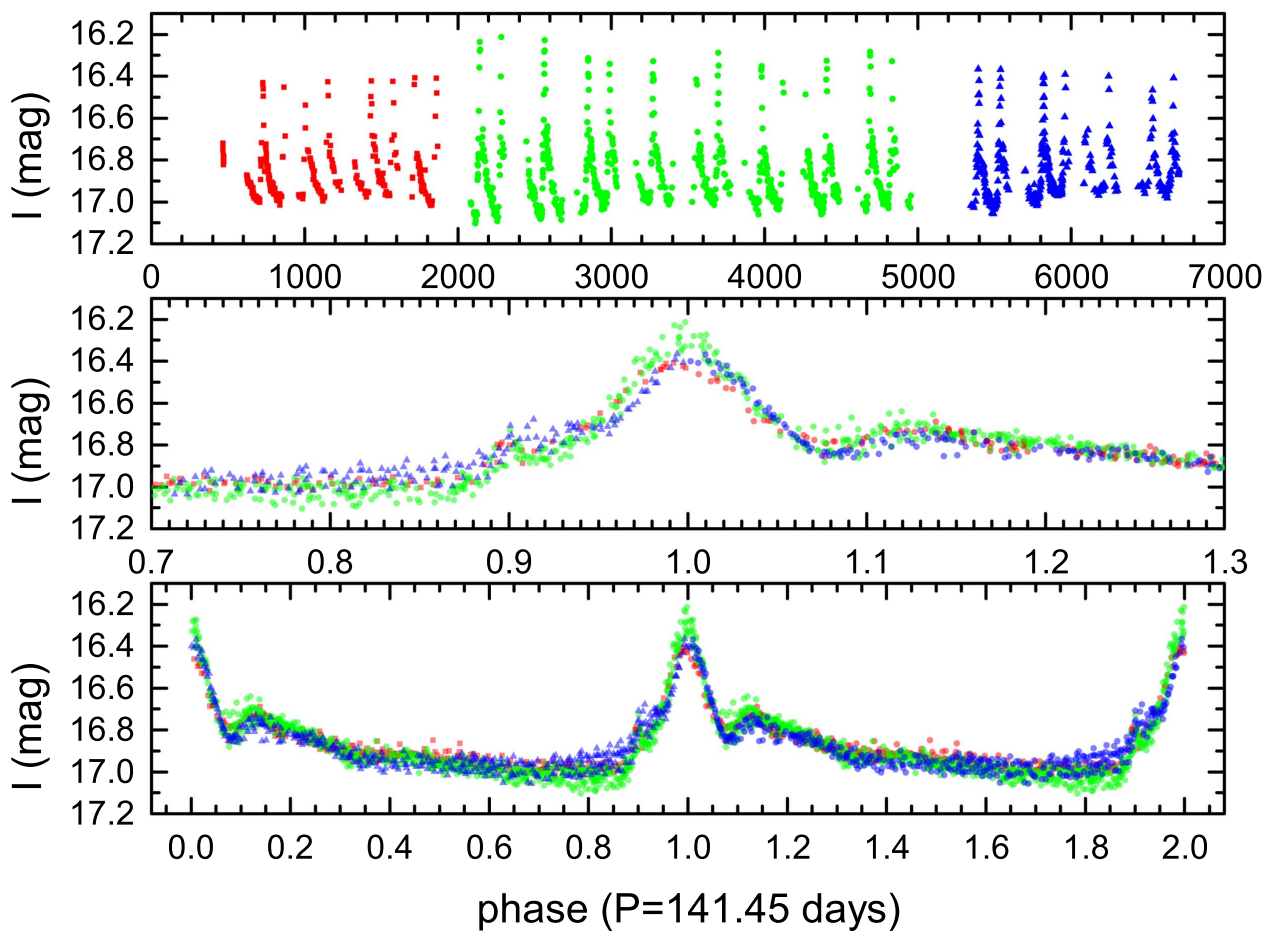

Figure 2. Figure 1: OGLE I-band light curve (top panel), phased with the particular period (141.45 days: middle and bottom panels). Colors red, green, and blue indicate magnitudes from photometric databases OGLE II, III, and IV, respectively.

recognizing spectra with minimum exposure time in the UV and optical ranges neglecting the infrared output and during the minimum (June observations) and maximum (July observations) of the photometric cycle described in Section 4. Slit widths of $1.0 \operatorname{arcsec}$ (blue) and $0.9 \operatorname{arcsec}$ (red) were used. The spectra were reduced using the X-SHOOTER pipeline, including bias removal, wavelength and flux calibration corrected by atmospheric differential refraction. Due to the crowdedness of the field, and to avoid including faint nearby stars in the spectrum, the sky was subtracted only in the visual range. One additional step was to remove the barycentric earth's velocity, hence the velocities given here are referred to the center of mass of the solar system. NIR observations are not considered in this study because of the extremely low signal-tonoise ratio of the spectra. Our spectroscopic observations are summarized in Table 2.

\section{Results}

\subsection{Analysis of the Light Curve}

We shifted OGLE-II magnitudes to fit the mean of OGLE-III and OGLE-IV data. 17.5 years of OGLE- $I$ and $V$-band photometry show light modulations similar to outbursts recurring with a period of 141.45 days (Figure 2). The period was obtained with the PDM task (Stellingwerf 1978) available in the NOAO software "Image Reduction and Analysis Facility" (IRAF ${ }^{12}$ ). These "outbursts" have a duty cycle of $20 \%$ and an amplitude much larger in $I$ than in the $V$ band; their shapes are almost constant, with a first excursion to a local maximum, followed by a small brightness decrease and then a second excursion to the maximum occurring around $I=16.4$. The system returns to minimum passing again by a dip and secondary peak as revealed in Figures 2 and 3. The maxima occur at the same time in both bands, but the minimum seems to occur earlier in $V$ band than in $I$ band (Figure 4). The $V-I$ color at minimum $(+0.12)$ is compatible with a F1 supergiant but at maximum the star is redder; $V-I=0.63$ indicates a F5 spectral type (see also Figure 2). In addition to the main light modulation, we find a very long cycle of timescale $T \sim 2500$ days, more evident in the lower envelope of the I-band light curve in the top panel of Figure 2. We find the following ephemerides for the maxima:

$$
H J D_{\max }=2450587.40+141.45 E .
$$

We searched for additional periodicities outside outburst, considering data only in the phase range $0.3-0.9$ and removing the $141.45 \mathrm{~d}$ periodicity, but no additional period was found.

\footnotetext{
12 IRAF is distributed by the National Optical Astronomy Observatory, which is operated by the Association of Universities for Research in Astronomy (AURA) under cooperative agreement with the National Science Foundation (http://iraf.noao.edu).
} 

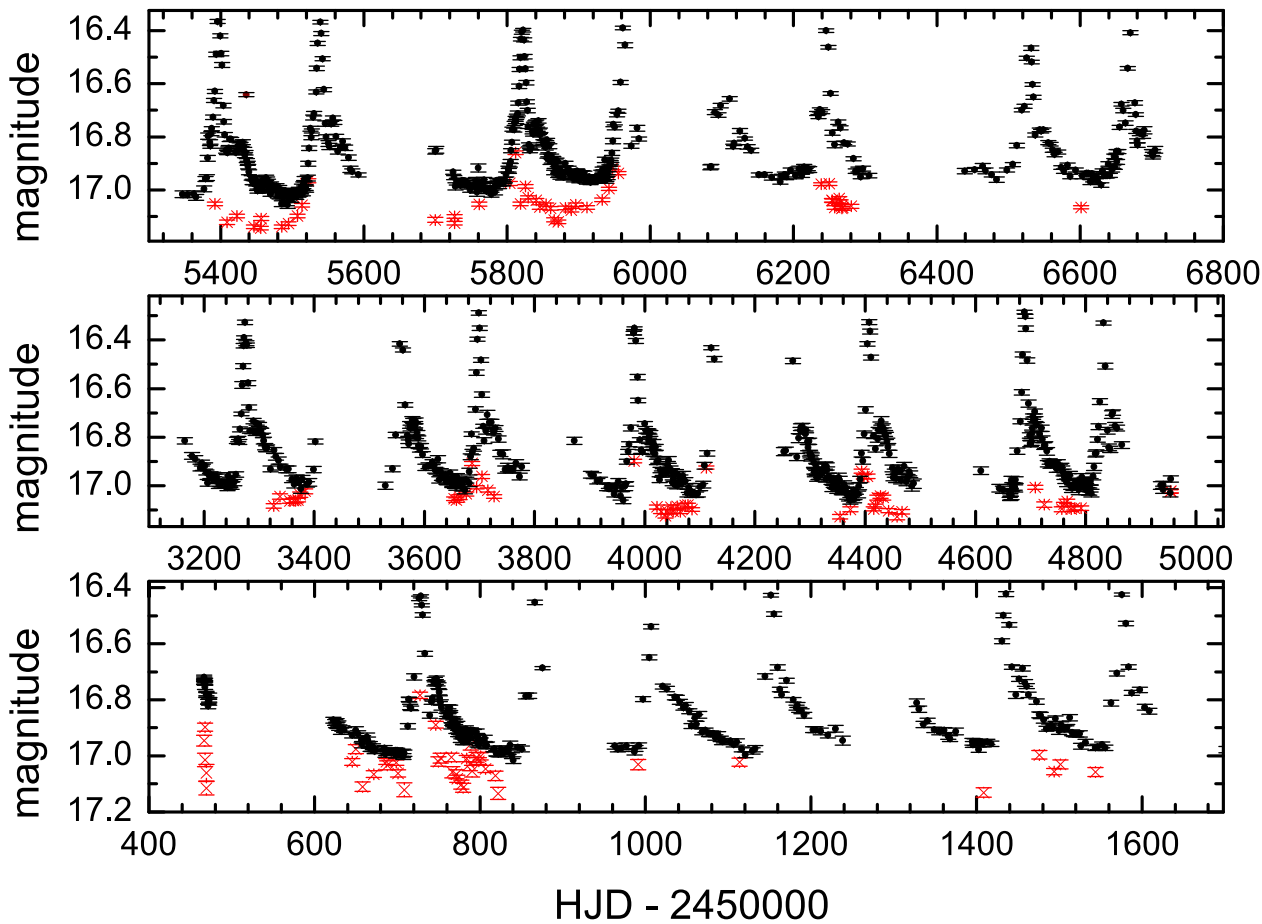

Figure 3. OGLE $I$-band (black dots) and $V$-band (red crosses) light curves at different epochs. Note the smaller amplitude variability in the $V$ band. (A color version of this figure is available in the online journal.)

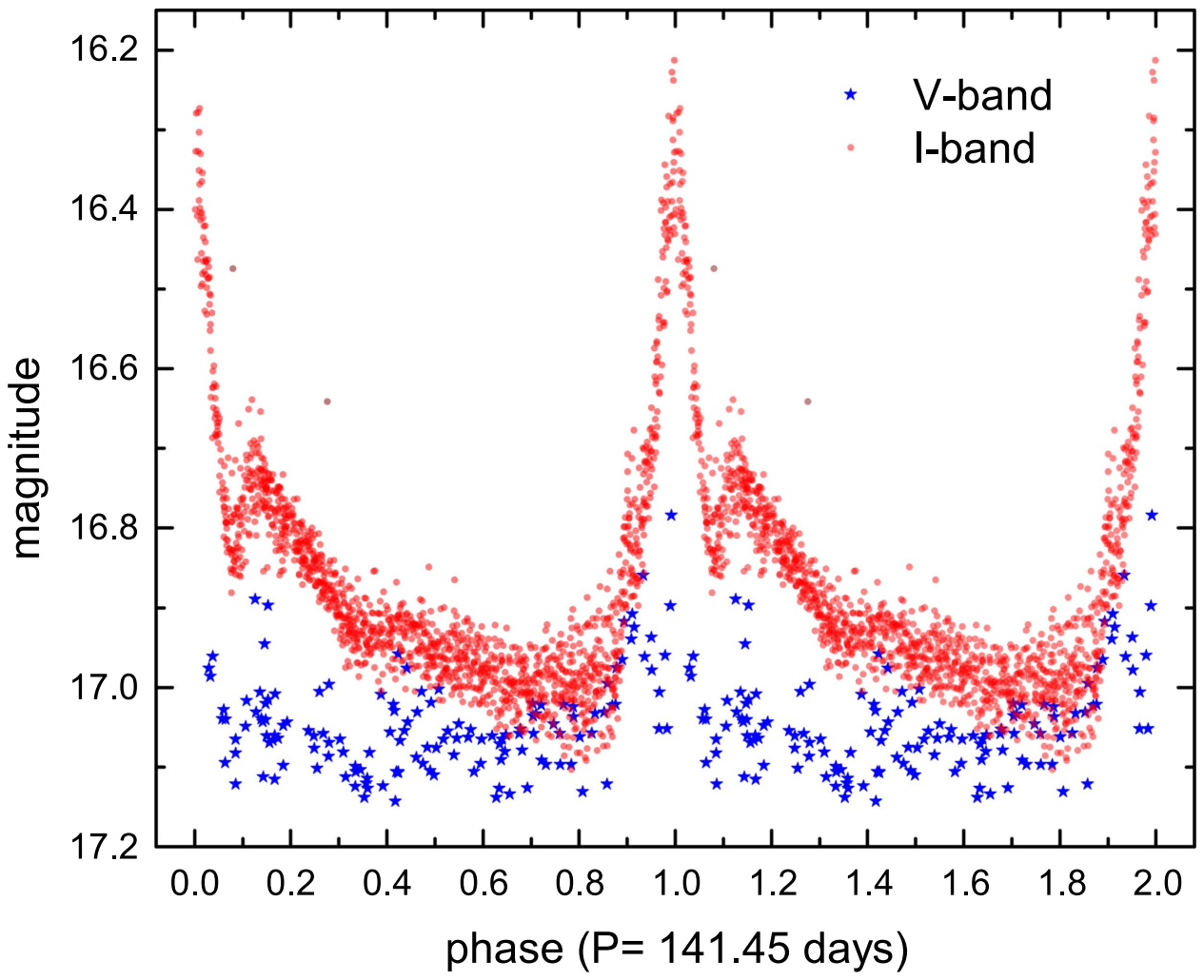

Figure 4. Comparison of $I$-band and $V$-band light curves during the 141.45 days cycle.

(A color version of this figure is available in the online journal.) 

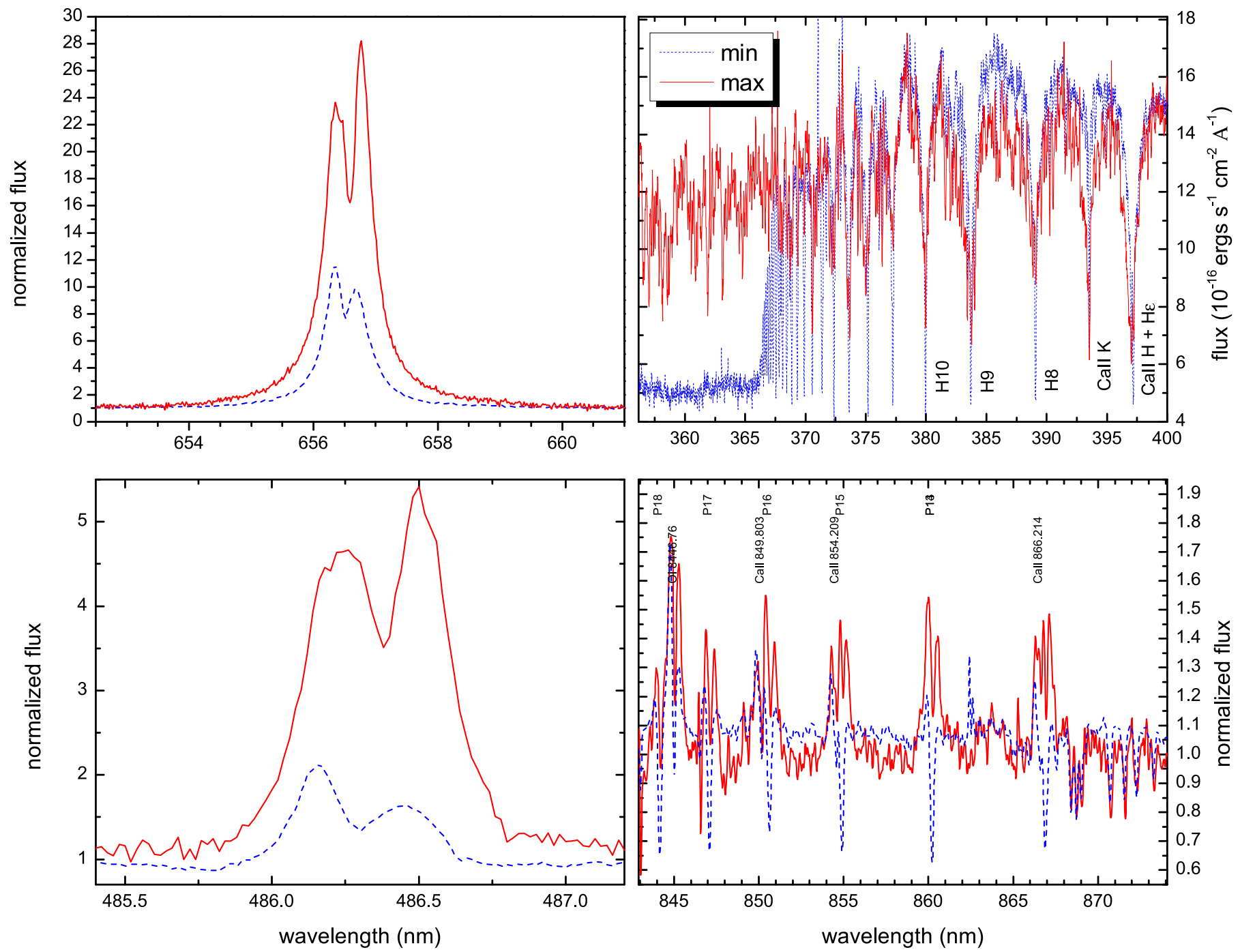

Figure 5. Comparison of spectral regions at maximum (solid red) and minimum (dashed blue). The flux calibration of the spectrum at maximum should not be trusted because of the contamination discussed in the text.

(A color version of this figure is available in the online journal.)

\subsection{Analysis of Spectroscopic Data}

\subsubsection{The Spectrum at Minimum}

The spectrum taken at minimum shows emission in $\mathrm{H} \alpha$ and He I $\lambda \lambda 5875 \AA$ and He I $\lambda \lambda 6678 \AA$ in absorption, revealing an early type, possibly B-type stellar component (Figure 5). The Paschen series is seen in absorption, along with double emission showing the violet peak larger than the red peak, i.e., $V>R$, where $V$ and $R$ refer to the respective peak intensities. We also find OI $\lambda \lambda 8446 \AA$ as single emission and some metallic double emissions. The spectrum shows a second Balmer discontinuity. This fact, along with the presence of H I emission and sharp absorptions of elements Cr II, Ti I, Ti II, Fe I, and Fe II reveals the presence of a circumstellar envelope.
The stellar parameters of the stars were obtained from direct measurements of the Balmer discontinuity. To this aim, we used the BCD method (Barbier \& Chalonge 1939; Chalonge \& Divan 1973) that has the advantage of studying the Balmer jump to recognize B-type stars with circumstellar envelopes due to the presence of a second component of the Balmer discontinuity (Divan 1979; Zorec \& Briot 1991; Aidelman et al. 2015, 2018). A strong second Balmer discontinuity is present in the spectrum taken at the minimum intensity of the light curve $\left(m_{v}=17.2\right)$. Based on the height of the Balmer jump $D_{\star}=0.17$ and its spectral position $\lambda_{1}=41 \AA$, we determined a spectral type $\mathrm{B} 2 / 3$ and temperature $T_{\text {eff }}=19000 \mathrm{~K}$ using the recent BCD calibration by Shokry et al. (2018). A spectral type B2/3 IV is consistent with $V=17.2$ in the SMC, as reveal the study of the star 
Publications of the Astronomical Society of the Pacific, 130:094204 (11pp), 2018 September

Mennickent et al.

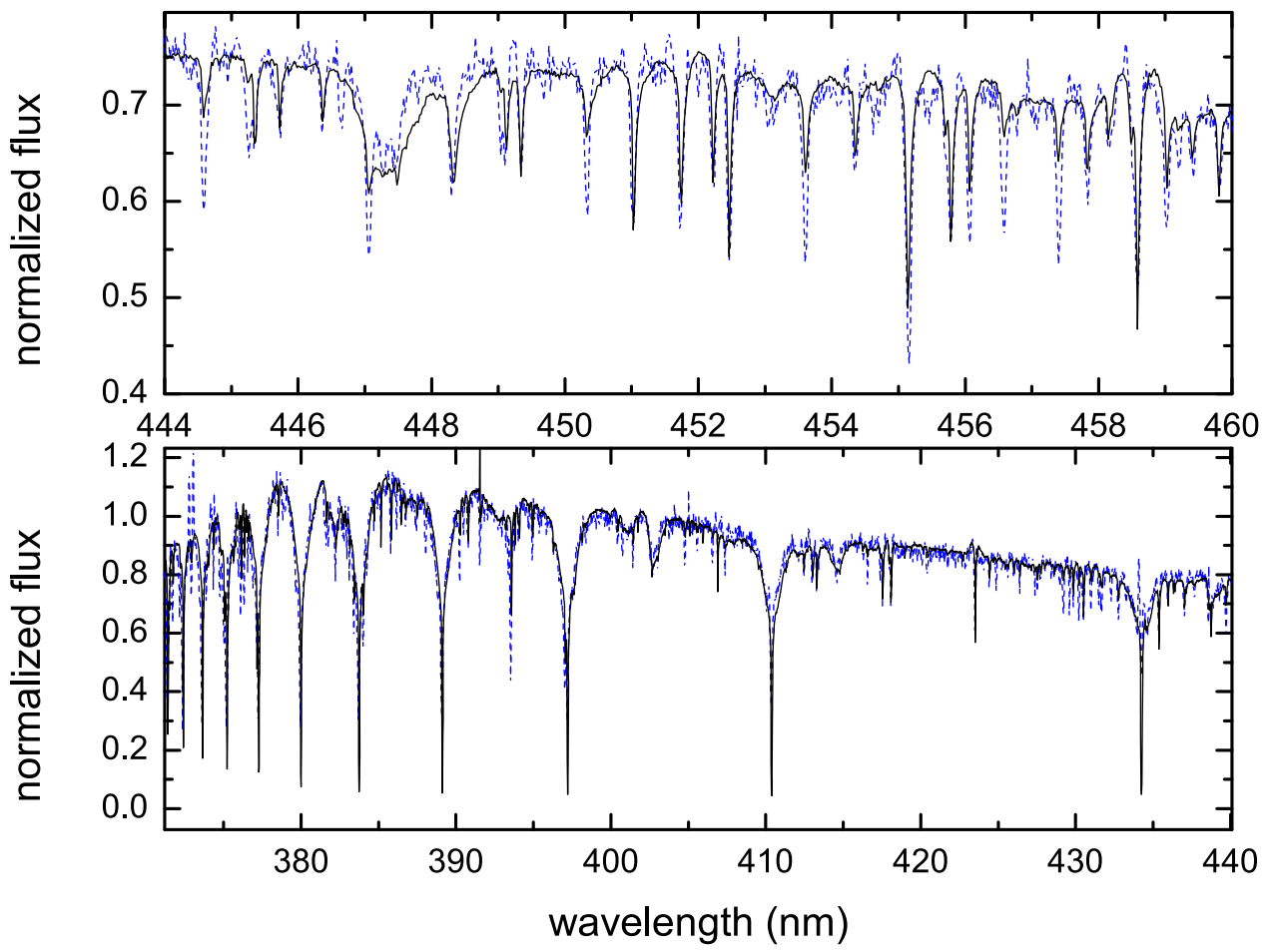

Figure 6. Comparison of the spectrum at minimum (dashed blue) with the spectrum of 48 Lib (HD 142983) which is classified B8 Ia/Iab. (A color version of this figure is available in the online journal.)

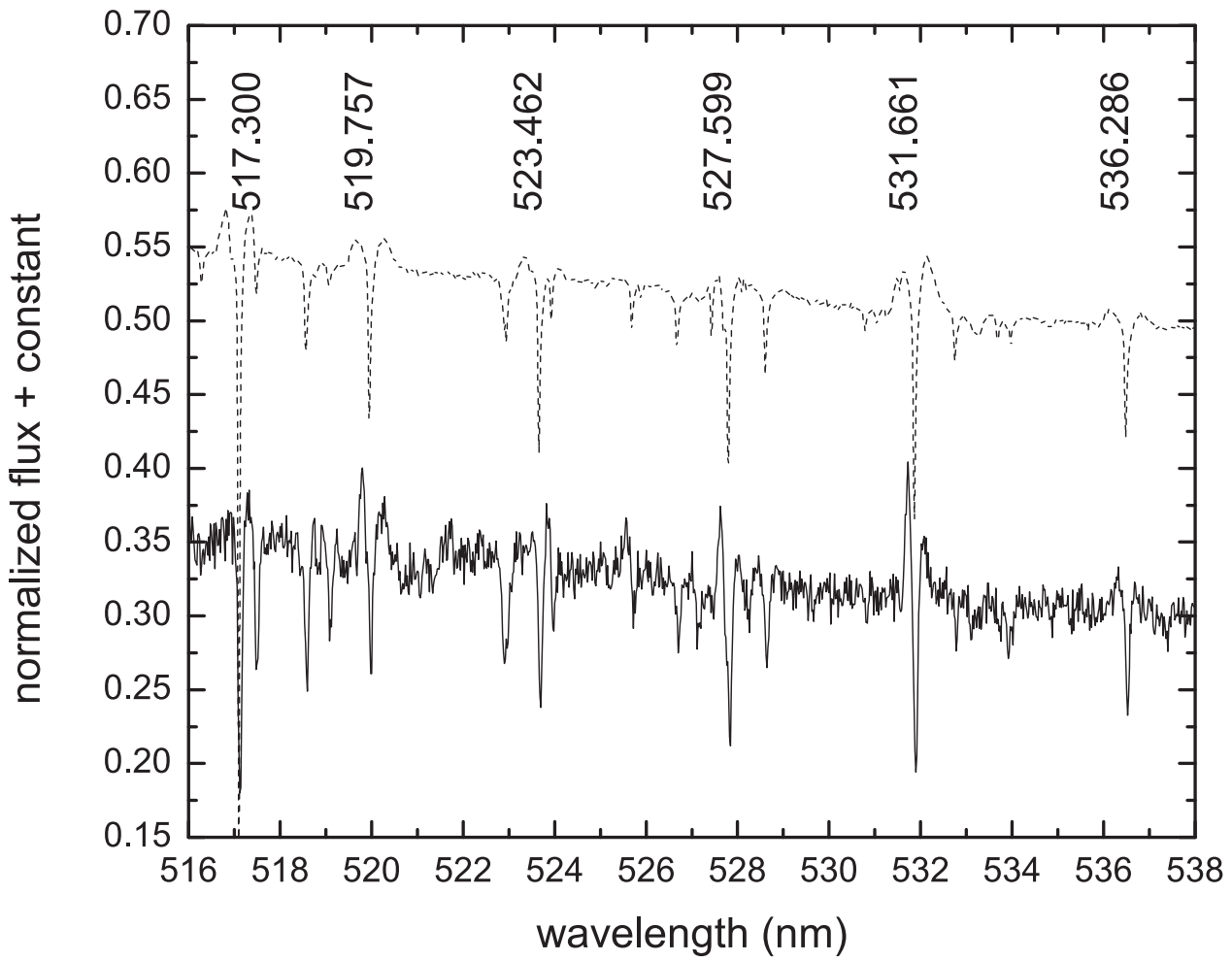

Figure 7. Some Fe II double-emission lines mostly showing the asymmetry $V>R$ (solid-line) and the comparison with the 48 Lib spectrum (dashed line). 


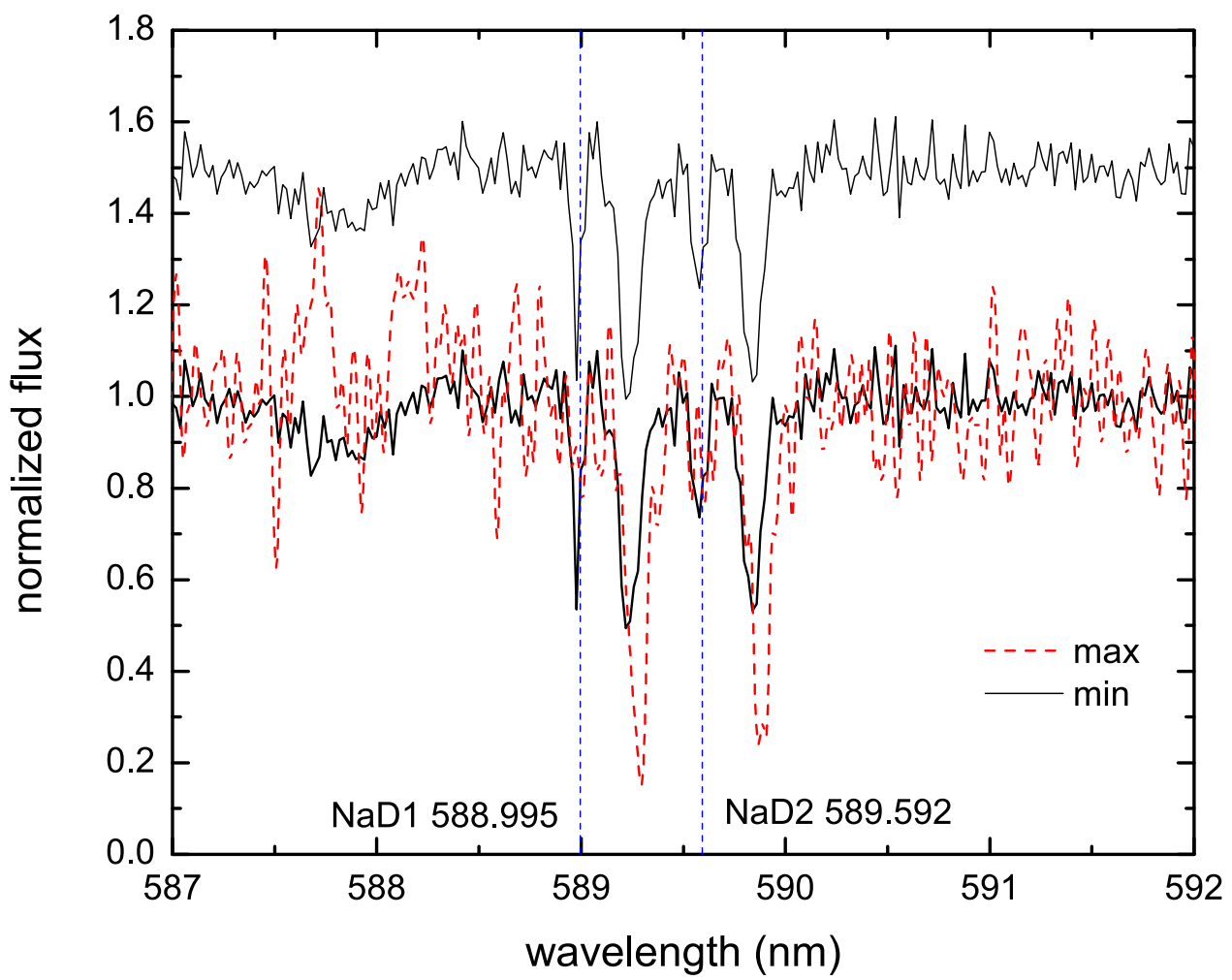

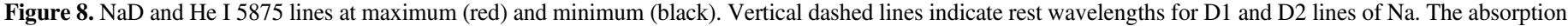
lines at the rest wavelengths are due to Galactic interstellar absorption. The spectrum at minimum is plotted twice and shifted for easier comparison.

(A color version of this figure is available in the online journal.)

Table 3

Average Measurements for Emission Lines and their Standard Deviations. The Methods of Barycenter and Central Minimum are Indicated for Radial Velocities with Typical Error $\pm 2 \mathrm{~km} / \mathrm{s}$

\begin{tabular}{|c|c|c|c|c|c|}
\hline Line & $\begin{array}{l}E W \\
(\AA)\end{array}$ & $\begin{array}{c}\Delta \lambda_{p} \\
\left(\mathrm{~km} \mathrm{~s}^{-1}\right)\end{array}$ & $\begin{array}{c}V / R \\
\text { or Note }\end{array}$ & $\begin{array}{l}\mathrm{RV}(\mathrm{bar}) \\
\left(\mathrm{km} \mathrm{s}^{-1}\right)\end{array}$ & $\begin{array}{l}\mathrm{RV} \text { (cen) } \\
\left(\mathrm{km} \mathrm{s}^{-1}\right)\end{array}$ \\
\hline \multicolumn{6}{|l|}{$\min$} \\
\hline $\mathrm{H} \alpha$ & $-102.8 \pm 0.5$ & $153 \pm 3$ & $1.19 \pm 0.01$ & 103 & 107 \\
\hline $\mathrm{H} \beta$ & $-5.0 \pm 0.1$ & $186 \pm 6$ & $1.76 \pm 0.01$ & 82 & 101 \\
\hline $\mathrm{H} \gamma$ & NA & $225 \pm 2$ & $V>R$ & 90 & 120 \\
\hline P14 & $0.50 \pm 0.05$ & $\ldots$ & $\ldots$ & $\ldots$ & 132 \\
\hline P17 & $\ldots$ & $\ldots$ & $\ldots$ & $\ldots$ & 132 \\
\hline \multicolumn{6}{|l|}{$\max$} \\
\hline $\mathrm{H} \alpha$ & $-269.7 \pm 3.0$ & $179 \pm 2$ & $0.83 \pm 0.04$ & 142 & 139 \\
\hline $\mathrm{H} \beta$ & $-24.0 \pm 0.2$ & $159 \pm 1$ & $0.83 \pm 0.02$ & 158 & 156 \\
\hline $\mathrm{H} \gamma$ & $-3.9 \pm 0.2$ & $188 \pm 5$ & $\mathrm{~V} \sim \mathrm{R}$ & 164 & 154 \\
\hline P14 & $-4.7 \pm 0.5$ & $198 \pm 5$ & $1.33 \pm 0.01$ & 135 & 148 \\
\hline P17 & $-5.0 \pm 0.5$ & $205 \pm 5$ & $1.20 \pm 0.01$ & 144 & 149 \\
\hline He I 5875 & $-1.0 \pm 0.5$ & $240 \pm 5$ & $\mathrm{~V} \sim \mathrm{R}$ & 219 & 180 \\
\hline
\end{tabular}

SMC_SC4 22859 with $V=17.1$ (Rímulo et al. 2018). These authors found eight solar masses in the middle of the MS for SMC_SC4 22859, and from our BCD analysis we get about 6.3 masses for our object, which is a reasonable discrepancy.

We also notice the similarity of the spectrum at minimum with the Galactic Be shell-star 48 Lib (HD 142983), classified as
B8 Ia/Iab in SIMBAD. The comparison with the metallic absorption lines of $48 \mathrm{Lib}$ produced in its envelope is specially interesting; the comparison spectrum is taken from the UVES-POP catalog ${ }^{13}$ (Figure 6). In the case of $48 \mathrm{Lib}$, this late

\footnotetext{
$\overline{13}$ http://www.eso.org/sci/observing/tools/uvespop.html
} 
Table 4

Radial Velocities of Some Absorption Lines at Maximum and Minimum

\begin{tabular}{lc}
\hline \hline Line & $\begin{array}{c}\mathrm{RV} \\
\left(\mathrm{km} \mathrm{s}^{-1}\right)\end{array}$ \\
\hline Min & $109 \pm 15^{*}$ \\
He I 5875 & $123.8 \pm 0.2$ \\
Si I 6347.1 & $127 \pm 3$ \\
He I 6678 & $122.7 \pm 0.2$ \\
Na I 5889.95 & $125.2 \pm 0.2$ \\
Na I 5895.92 & $125 \pm 2$ \\
\hline Mean (min) & \\
excluding ${ }^{*}$ & \\
\hline Max & $145.8 \pm 0.2$ \\
Na I 5889.95 & $147.7 \pm 0.2$ \\
Na I 5895.92 & $147.0 \pm 0.2$ \\
Si I 6347.1 & $147 \pm 1$ \\
\hline Mean (max) & \\
\hline
\end{tabular}

Table 5

Radial Velocities and Peak Separation for Double-emission Fe II Lines Observed During Minimum

\begin{tabular}{lcccc}
\hline \hline $\begin{array}{l}\text { (lab) } \\
(\mathrm{A})\end{array}$ & $\begin{array}{c}\text { Abs } \\
\left(\mathrm{km} \mathrm{s}^{-1}\right)\end{array}$ & $\begin{array}{c}\text { Blue-em } \\
\left(\mathrm{km} \mathrm{s}^{-1}\right)\end{array}$ & $\begin{array}{c}\text { Red-em } \\
\left(\mathrm{km} \mathrm{s}^{-1}\right)\end{array}$ & $\begin{array}{c}\Delta \lambda_{p} \\
\left(\mathrm{~km} \mathrm{~s}^{-1}\right)\end{array}$ \\
\hline 4233.167 & 118.9 & 16.2 & 222.3 & 206.1 \\
4583.829 & 108.9 & -0.4 & 241.0 & 241.3 \\
4629.336 & 116.9 & 20.4 & 234.8 & 214.4 \\
4924.043 & 110.1 & 4.1 & $\ldots$ & $\ldots$ \\
5018.434 & 116.7 & 11.0 & 213.5 & 202.5 \\
5173.002 & 103.7 & -8.1 & $\ldots$ & $\ldots$ \\
5197.569 & 123.6 & 10.5 & 257.4 & 246.9 \\
5275.994 & 123.0 & -0.9 & $\ldots$ & $\ldots$ \\
$5316.609 / 777$ & 118.6 & 17.1 & 209.4 & 192.3 \\
5362.864 & 119.1 & 4.5 & $\ldots$ & $\ldots$ \\
\hline Average & 115.9 & 7.5 & 229.7 & 217.2 \\
Std & 6.4 & 9.2 & 18.2 & 22.0 \\
\hline
\end{tabular}

classification is purely due to the shell, the actual star has a temperature corresponding to about B3 (Silaj et al. 2016). The supergiant classification is also only because of the shell, because it has a very strong $\mathrm{V} / \mathrm{R}$ cycle (relative intensity between emission peaks) that can look like a P Cyg wind profile at times (Silaj et al. 2016). Some Fe II double-emission lines with $V>R$ are shown in Figure 7 for comparison with the $48 \mathrm{Lib}$ case.

\subsubsection{The Spectrum at Maximum}

We observe much weaker Balmer absorptions than in minimum and $\mathrm{He} \mathrm{I} \lambda \lambda 5875 \AA$ appears as double emission with
Table 6

Radial Velocities of Absorption Lines Observed During Minimum. The Observed Wavelengths are not Barycentric Corrected (the Velocities do)

\begin{tabular}{|c|c|c|}
\hline $\begin{array}{l}\lambda \text { (lab) } \\
(\AA)\end{array}$ & $\begin{array}{c}\lambda(\text { obs }) \\
(\AA)\end{array}$ & $\begin{array}{c}\mathrm{RV} \\
\left(\mathrm{km} \mathrm{s}^{-1}\right)\end{array}$ \\
\hline \multicolumn{3}{|l|}{ H I } \\
\hline 3663.406 & 3664.943 & 125.8 \\
\hline 3664.679 & 3666.252 & 128.7 \\
\hline 3666.097 & 3667.666 & 128.3 \\
\hline 3667.684 & 3669.212 & 124.9 \\
\hline 3669.466 & 3671.035 & 128.2 \\
\hline 3671.478 & 3673.019 & 125.8 \\
\hline 3673.761 & 3675.316 & 126.9 \\
\hline 3676.365 & 3677.916 & 126.5 \\
\hline 3679.355 & 3680.980 & 132.4 \\
\hline 3682.810 & 3684.333 & 124.0 \\
\hline 3686.833 & 3688.414 & 128.6 \\
\hline 3691.557 & 3693.054 & 121.6 \\
\hline 3697.154 & 3698.736 & 128.3 \\
\hline 3703.855 & 3705.404 & 125.4 \\
\hline 3711.973 & 3713.678 & 137.7 \\
\hline 3721.940 & 3723.517 & 127.0 \\
\hline 3734.370 & 3735.981 & 129.3 \\
\hline 3750.154 & 3751.652 & 119.8 \\
\hline 3770.632 & 3772.090 & 115.9 \\
\hline 3797.900 & 3799.448 & 122.2 \\
\hline 3835.386 & 3836.957 & 122.8 \\
\hline 3889.051 & 3890.670 & 124.8 \\
\hline 4101.737 & 4103.427 & 123.5 \\
\hline \multicolumn{3}{|l|}{ Fe I } \\
\hline 4307.902 & 4309.796 & 120.9 \\
\hline 4404.750 & 4406.709 & 122.4 \\
\hline \multicolumn{3}{|l|}{$\mathrm{Fe}$ II } \\
\hline 4508.288 & 4510.250 & 119.6 \\
\hline 4520.224 & 4522.205 & 120.5 \\
\hline 4522.634 & 4524.651 & 122.8 \\
\hline 4555.893 & 4557.738 & 110.5 \\
\hline 4848.235 & 4850.341 & 119.3 \\
\hline 5284.109 & 5286.394 & 118.7 \\
\hline \multicolumn{3}{|l|}{ Ti II } \\
\hline 4320.960 & 4322.861 & 121.0 \\
\hline 4443.798 & 4445.904 & 131.2 \\
\hline 4464.450 & 4466.479 & 125.3 \\
\hline 4501.272 & 4503.293 & 123.7 \\
\hline 4533.966 & 4536.075 & 128.5 \\
\hline 4911.193 & 4913.348 & 120.6 \\
\hline 4563.761 & 4565.828 & 124.9 \\
\hline 4571.969 & 4574.048 & 125.4 \\
\hline \multicolumn{3}{|l|}{ Mg II } \\
\hline 4481.226 & 4483.126 & 116.2 \\
\hline \multicolumn{3}{|l|}{$\mathrm{Cr}$ II } \\
\hline 4824.127 & 4826.172 & 116.2 \\
\hline 4876.440 & 4878.554 & 119.1 \\
\hline Average \pm std & $\ldots$ & $123.9 \pm 5.0$ \\
\hline
\end{tabular}




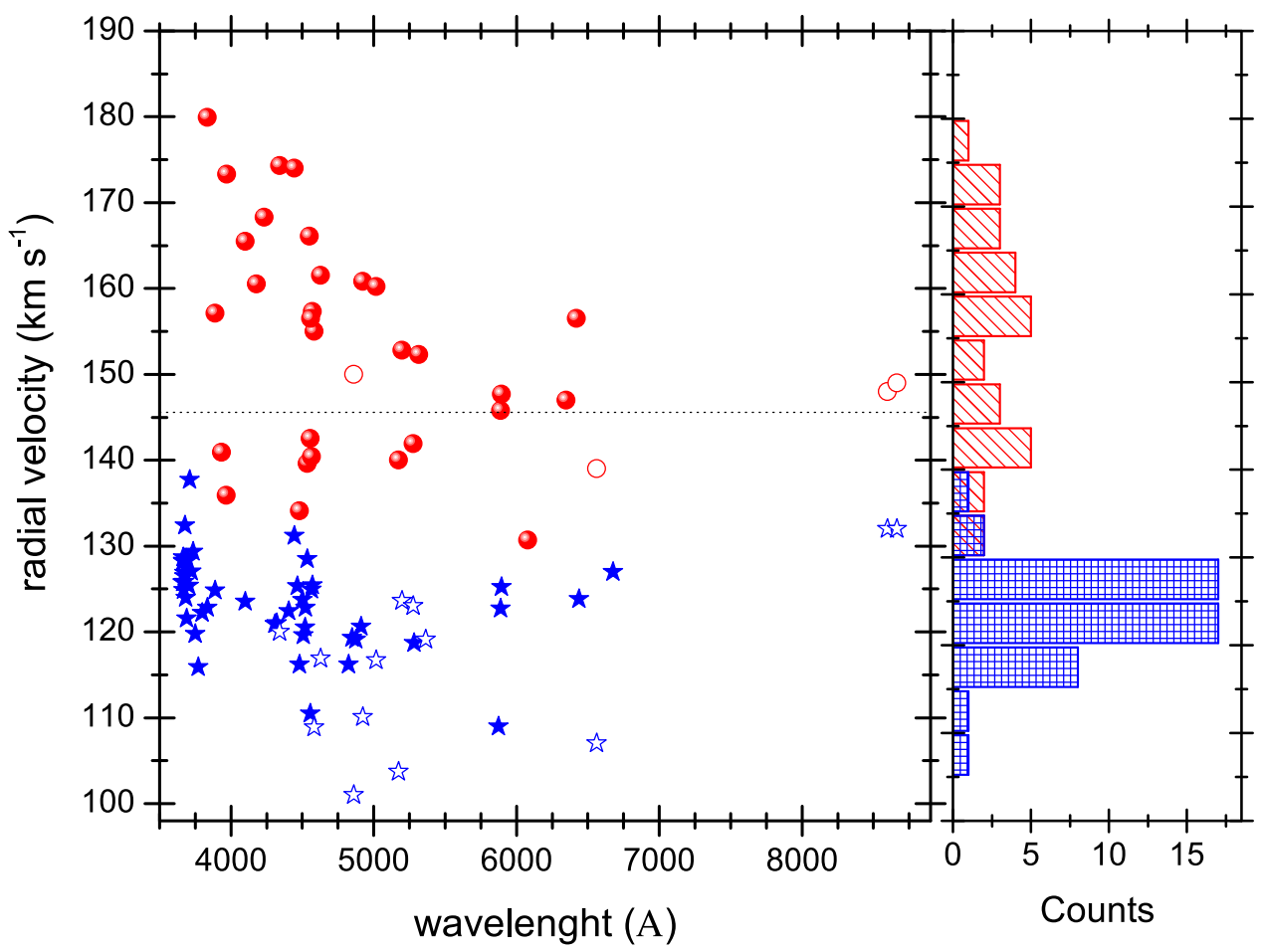

Figure 9. Radial velocities of absorption lines at maximum (red bullets) and minimum (blue stars) and the respective histograms. Open symbols indicating central absorption radial velocity of double-emission lines are also shown. The heliocentric velocity of the SMC, $145.6 \mathrm{~km} \mathrm{~s}^{-1}$ (McConnachie 2012) is indicated as a dotted line. (A color version of this figure is available in the online journal.)

peak separation of $240 \mathrm{~km} \mathrm{~s}^{-1}$ (Figure 8). As the spectrum is quite noisy in that region, and other helium lines as He I $\lambda \lambda 4471 \AA$ do not show emission, this detection should be considered as tentative only. Interestingly, as shown in Figure 5, double emission is detected with confidence in the infrared Calcium triplet. At this stage, the Balmer/Paschen double emissions are in general larger and without the deep absorption cores observed during minimum. The circumstellar metallic absorption lines still are visible during maximum. An additional set of metallic lines appears, which is not observed at minimum; this is specially evident in the blue spectral region. Since they span the whole spectrum in the spatial direction at both sides of the stellar profile, this background metallic line spectrum probably arises from reflected moonlight. In spite of this contamination, a set of lines characterized by radial velocities displaced by about $+140 \mathrm{~km} / \mathrm{s}$ from the background spectrum are present. Their velocities are compatible with an origin in the SMC and we assumed they are formed in the system under study.

At maximum, we also find a stronger stellar $\mathrm{NaD}$ doublet (Figure 8). The system $\mathrm{NaD}$ lines are clearly distinguished from the sharp Galactic interstellar $\mathrm{NaD}$ lines, that are more clearly visible at minimum. The SMC interstellar components are expected much weaker than the Galactic ones and are obviously masked by the system components, which are much stronger than expect for a B-type star, and are probably formed in the circumstellar medium. The increasing strength at maximum, along with the infrared CaII triplet emission suggest an accretion phenomenon and a formation in a mass stream as we will discuss in Section 5.

\subsubsection{General Spectroscopic Analysis}

Average properties for Balmer and Paschen emission lines are presented in Table 3, including the ratio between the violet and red peak intensity relative to the normalized continuum $V / R \equiv\left(I_{V}-1\right) /\left(I_{R}-1\right)$. The peak separation increases with the Balmer series order. This gradient, and the existence of double emission, are typical signatures of a Keplerian Be star disk. Equivalent widths $(E W)$ were measured between the adjacent continuum of the $\mathrm{H} \alpha$ line and tracing a line at the base of the other emission lines.

In general, we notice larger $\mathrm{H} \mathrm{I}$ emission at maximum, along with changes in $\mathrm{HI}$ line shapes. At minimum $V>R$ and at maximum $V<R$ in Balmer lines. However, in Paschen lines $V>R$ in both epochs. At maximum, we also observe Ca II lines as double emissions. While the $\mathrm{H} \alpha$ peak separation increases at maximum the opposite is observed in $\mathrm{H} \beta . \mathrm{H} \beta$ remains almost of the same strength relative to the continuum at both epochs.

Radial velocities for $\mathrm{He}$ I, Si I and $\mathrm{NaD}$ absorption lines are given in Table 4, for Fe II emission lines (along with peak 
Table 7

Radial Velocities of Absorption Lines Observed during Maximum

\begin{tabular}{|c|c|c|}
\hline $\begin{array}{l}\lambda(\mathrm{lab}) \\
(\AA)\end{array}$ & $\begin{array}{c}\lambda(\text { obs }) \\
(\AA)\end{array}$ & $\begin{array}{c}\mathrm{RV} \\
\left(\mathrm{km} \mathrm{s}^{-1}\right)\end{array}$ \\
\hline \multicolumn{3}{|l|}{$\mathrm{H}$} \\
\hline 4340.472 & 4342.93 & 174.3 \\
\hline 4101.737 & 4103.94 & 165.5 \\
\hline 3970.075 & 3972.31 & 173.3 \\
\hline 3889.051 & 3891.03 & 157.1 \\
\hline 3835.386 & 3837.63 & 179.9 \\
\hline \multicolumn{3}{|l|}{ Fe II } \\
\hline 4178.855 & 4181.03 & 160.5 \\
\hline 4233.167 & 4235.48 & 168.3 \\
\hline 4549.467 & 4551.92 & 166.1 \\
\hline 4555.893 & 4557.99 & 142.5 \\
\hline 4583.829 & 4586.13 & 155.0 \\
\hline 4629.336 & 4631.76 & 161.5 \\
\hline 4924.043 & 4926.61 & 160.8 \\
\hline 5018.434 & 5021.04 & 160.2 \\
\hline 5173.002 & 5175.34 & 140.0 \\
\hline 5197.569 & 5200.14 & 152.8 \\
\hline 5275.994 & 5278.41 & 141.9 \\
\hline 5316.609 & 5319.23 & 152.3 \\
\hline \multicolumn{3}{|l|}{ Ca II } \\
\hline 3933.660 & 3935.45 & 140.9 \\
\hline 3968.470 & 3970.21 & 135.9 \\
\hline \multicolumn{3}{|l|}{$\mathrm{Mg}$ II } \\
\hline 4481.226 & 4483.17 & 134.1 \\
\hline \multicolumn{3}{|l|}{ Ti II } \\
\hline 4443.798 & 4446.31 & 174.0 \\
\hline 4533.966 & 4536.01 & 139.6 \\
\hline 4563.761 & 4565.83 & 140.4 \\
\hline 4571.969 & 4574.3 & 157.3 \\
\hline \multicolumn{3}{|l|}{ Cr II } \\
\hline 4558.659 & 4560.97 & 156.5 \\
\hline \multicolumn{3}{|l|}{ Fe I } \\
\hline 6078.500 & 6081.06 & 130.7 \\
\hline 6419.980 & 6717.13 & 156.5 \\
\hline Average & $\ldots$ & $154.0 \pm 13.0$ \\
\hline
\end{tabular}

separation) are given in Table 5 and for $\mathrm{HI}$ and metallic absorption lines are given in Table 6. Radial velocities of absorption lines observed during maximum are given in Table 7. At maximum, we observe a likely bimodal distribution of the radial velocities suggesting two components, and at minimum only one component is clearly observed and separated from the velocities at maximum (Figure 9); these facts suggest furthermore that the system is a binary star.

\section{Discussion}

At minimum, the B-type component dominates the spectrum and the set of radial velocities of the envelope roughly matches those helium-line velocities associated to the star (Tables 4 and 6). This suggests that a circumstellar envelope surrounds the B-type star. The double-peak structure of the H I emission and the presence of $\mathrm{V} / \mathrm{R}$ variability suggests a disc-shaped envelope. $\mathrm{V} / \mathrm{R}$ variability is usually observed in Be stars and interpreted as oscillations of density enhancements and explained by the one-armed oscillation theory (Okazaki 1991). Furthermore, quasi-cyclic photometric variability in timescales of thousands of days, as observed in this system, has been sometimes reported in Be stars. We notice that the observed long-cycle period of 2500 days (6.8 years) is close to the average of the $\mathrm{V} / \mathrm{R}$ variability timescale, viz. 7 years (Mennickent \& Vogt 1991). The above suggests a Be star nature for this system. The Classical Be stars are rapidly rotating non-supergiants B-type stars that show or have shown Balmer line emission in the past (Rivinius et al. 2013). The emission is formed in a circumstellar disk by electron excitation and subsequent cascade recombination in the circumstellar material, mostly neutral hydrogen. On the observational side, the system show similarities to the Be star ABE-A01, showing outbursts with a quasi-period of 91.23 days (Labadie-Bartz et al. 2018).

At maximum we observe two sets of radial velocities clearly separated and different from the radial velocities observed at minimum. This fact, along with the shape of the light curve, might indicate that the system is a binary of orbital period 141.45 days with a dense circumstellar disc and an eccentric orbit. Furthermore, we notice that at maximum the $\mathrm{H} \alpha$ and Paschen emission are larger and the $\mathrm{NaD}$ lines are stronger, indicating possibly a mass transfer episode in an eccentric binary system when the unseen secondary star passes at periastron, overflowing its Roche lobe and depositing material onto the Be star disc. Supporting this accretion scenario, the detection of the infrared Ca II triplet in emission in Be stars has been interpreted in terms of binarity and stream accretion (Koubský et al. 2012; Shokry et al. 2018). However, at the present stage, we cannot discard that during passage by periastron an increased injection of mass might occur from the surface of the Be star into the disk, producing the observed brightenings (ejection scenario).

Future challengers include the determination of the nature of the unseen secondary star and the determination of the orbital parameters, including an explanation for the possible eccentricity. The secondary is probably not a compact object, because of the lack of X-ray and high-excitation lines; also because of the outbursts are of low amplitude, reflecting-in the accretion scenario-the fall of material into a shallow gravitational potential. On the other hand, the secondary star should be much less luminous that the Be star to remain undetected in the spectrum. This suggests a lower temperature main-sequence secondary star. In the ejection scenario, its mass should be not so low in order to perturb appreciably the gravitationally bounded material in the surface of the Be star. 
Another possibility is a low-mass OB subdwarf secondary, as seen in HR 2142 (Peters et al. 2016) and other recently found $\mathrm{Be}+\mathrm{sdO}$ Galactic binaries (Wang et al. 2018). These relatively hot, but very faint, objects were detected only in combined spectra with very high signal-to-noise obtained in the ultraviolet spectral region by the International Ultraviolet Explorer satellite.

\section{Conclusions}

Based on the study of 17.5 years of $I$ - and $V$-band OGLE photometry and new X-SHOOTER spectra we find that OGLEJ005039.05-725751.4 is very likely a binary consisting of a $\mathrm{Be}$ star in an eccentric orbit with orbital period 141.45 days. We also detect a long cycle of 2500 days in the photometric data set. The strange character of the light curve might be explained by periodic mass transfer in a complex binary system as happens in certain types of cataclysmic variables. This is supported by our finding that at maximum the $\mathrm{H} \alpha$ emission is larger, the infrared Calcium triplet is seen in emission and the $\mathrm{NaD}$ lines are stronger. A detailed explanation of the system is beyond the capabilities of the available data. Future plans include the acquisition of time resolved spectroscopy to resolve the binary orbit and enlighten the brightening and line emission enhancement episode.

The authors thank the anonymous referee for providing useful comments that improved the first version of this manuscript. This research has made use of the SIMBAD database, operated at CDS, Strasbourg, France. R.E.M. acknowledges support by VRID-Enlace 218.016.004-1.0, VRID-Enlace 216.016.002-1.0 and the BASAL Centro de Astrofísica y Tecnologías Afines (CATA) AFB-170002. L.C. acknowledges financial support from the Agencia de Promoción Científica y Tecnológica (Préstamo BID PICT 2016/ 1971), CONICET (PIP 0177), and the Universidad Nacional de La Plata (Programa de Incentivos G11/137), Argentina. L.C. thanks also support from the project CONICYT + PAI/ Atracción de capital humano avanzado del extranjero (folio PAI80160057). The OGLE project has received funding from the Polish National Science Centre grant MAESTRO no. 2014/ 14/A/ST9/00121.

\section{References}

Abt, H. A., Levato, H., \& Grosso, M. 2002, ApJ, 573, 359

Aidelman, Y., Cidale, L. S., Zorec, J., \& Panei, J. A. 2015, A\&A, 577, A45 Aidelman, Y., Cidale, L. S., Zorec, J., \& Panei, J. A. 2018, A\&A, 610, A30 Barbier, D., \& Chalonge, D. 1939, ApJ, 90, 627

Bica, E., \& Dutra, C. M. 2000, AJ, 119, 1214

Chalonge, D., \& Divan, L. 1973, A\&A, 23, 69

Divan, L. 1979, RA, 9, 247

Koubský, P., Kotková, L., Votruba, V., et al. 2012, arXiv:1205.2259

Labadie-Bartz, J., Chojnowski, S. D., Whelan, D. G., et al. 2018, AJ, 155, 53

Martayan, C., Baade, D., \& Fabregat, J. 2010, A\&A, 509, A11

McConnachie, A. W. 2012, AJ, 144, 4

Mennickent, R. E., Pietrzyński, G., Gieren, W., \& Szewczyk, O. 2002, A\&A, 393, 887

Mennickent, R. E., \& Vogt, N. 1991, A\&A, 241, 159

Okazaki, A. T. 1991, PASJ, 43, 75

Peters, G. J., Wang, L., Gies, D. R., \& Grundstrom, E. D. 2016, ApJ, 828, 47

Rímulo, L. R., Carciofi, A. C., Vieira, R. G., et al. 2018, MNRAS, 476, 3555

Rivinius, T., Carciofi, A. C., \& Martayan, C. 2013, A\&ARv, 21, 69

Shokry, A., Rivinius, Th., Mehner, A., et al. 2018, A\&A, 609, A108

Silaj, J., Jones, C. E., Carciofi, A. C., et al. 2016, ApJ, 826, 81

Stellingwerf, R. F. 1978, ApJ, 224, 953

Szymanski, M. K. 2005, AcA, 55, 43

Udalski, A., Szymański, M. K., \& Szymański, G. 2015, AcA, 65, 1

Wang, L., Gies, D. R., \& Peters, G. J. 2018, ApJ, 853, 156

Zorec, J., \& Briot, D. 1991, A\&A, 245, 150 